Background Mycoplasma genitalium (MG) is a sexually transmitted bacterial pathogen implicated in urogenital disease of both men and women. Of importance, MG is associated with upper reproductive tract sequelae in women such as pelvic inflammatory disease (PID). In earlier studies, we have defined pig-tailed macaques as a suitable animal model for chronic lower reproductive tract infection, though only a subset of the primates remained persistently infected after cervical inoculation. Building on our previous findings, we refined our inoculation methods to increase infection rates, extended the course of infection, and measured the ascension of MG to the upper reproductive tract to model PID.

Methods Three female pig-tailed macaques were infected with an $\mathrm{MG}$ inoculum $\left(2 \times 10^{9}\right.$ genomes) prepared to minimize cell aggregates. Lower reproductive tract specimens were collected over 18 weeks. MG infection status was determined by culture in SP4 broth, Vero cell co-cultures, and qPCR. Humoral and cervical antibody responses to MG whole-cell lysates were analyzed through immunoblots.

Results All three primates were persistently infected, one for 8 weeks and two for $>14$ weeks. Primates that cleared the infection were re-infected to promote ascension to the upper reproductive tract. Humoral and cervical antibodies to the immunodominant $\mathrm{MgpB}$ and $\mathrm{MgpC}$ adherence proteins were detected after three weeks but were unable to clear MG infection.

Conclusion In prior experiments, MG persisted in only 4/9. In this pilot study, all three primates maintained persistent infection. MG-specific antibodies detected from both serum and cervical specimens will provide an important resource for the optimization of serologic tests that may elucidate immune mechanism of evasion in the genital tract. Future experiments will discern MG pathogenesis, including upper reproductive tract sequelae, as well host-pathogen interactions in a relevant animal model.

\section{P360 SINGLE GENE TARGETED NANOPORE SEQUENCING FOR SIMULTANEOUS IDENTIFICATION AND ANTIMICROBIAL RESISTANCE DETECTION OF SEXUALLY TRANSMITTED INFECTIONS}

'L Zhou, ${ }^{2} \mathrm{~A}$ Lopez Rodas, ${ }^{2} \mathrm{~L}$ LLangarí-Arizo, ${ }^{2,3} \mathrm{~N}$ Romero-Sandoval, ${ }^{1,2} \mathrm{P}$ Cooper, ${ }^{1} \mathrm{~S} S \mathrm{Sadiq}$ * ${ }^{1}$ Applied Diagnostic Research and Evaluation Unit, Institute for Infection and Immunity, St George's, University of London, London, UK; ${ }^{2}$ School of Medicine, Faculty of Health and Life Sciences, Universidad Internacional del Ecuador, Quito, Ecuador; ${ }^{3}$ Grups de Recerca d'Amèrica i Àfrica Llatines, GRAAL, Barcelona, Spain

10.1136/sextrans-2021-sti.404

Background World Health Organization guidelines to treat sexually transmitted infections (STIs) with the right antibiotic, at the right dose and the right time are undermined by spread of antimicrobial resistance (AMR). Accurate and rapid diagnostics simultaneously predicting antibiotic susceptibility and diagnosis may help address this challenge. We report early data from a novel approach using single gene targeted nanopore sequencing for simultaneous STI identification and AMR detection among female sex workers (FSWs) in Ecuador.

Methods Real-time PCR (RT-PCR) was performed on vulvovaginal swab samples from 200 FSWs to identify Neisseria gonorrhoeae (NG), Chlamydia trachomatis (CT), Mycoplasma genitalium (MG) and Trichomonas vaginalis (TV) infections. Samples positive and negative (controls) for these STIs were amplified and barcoded by PCR, targeting genes: gyrA (NG), ntr6 (TV) and 23S rRNA (MG), which confer resistance to fluoroquinolones, metronidazole and macrolides respectively, and omp1 (CT). A DNA library, constructed from a pool of these barcoded samples, was sequenced on Oxford Nanopore Technologies (ONT) MinION sequencer controlled by the smartphone operated 'MinIT' hand-held processor. Data were analysed using ONT AMR workflow, with manual BLAST confirmation.

Results Among PCR positives analysed, 25/26 of CT, NG or MG infections were clearly distinguishable from controls using sequence read counts, but discrimination of TV positives from controls was challenging as many had low pathogen loads (RT-PCR: cycle threshold $>35$ ) with associated low sequence read counts. Manual BLAST confirmed 3/3 NG, 2/10 TV, and 0/11 MG had fluoroquinolone, metronidazole and macrolide resistance-associated mutations respectively. Overall library preparation and sequencing time took approximately about five hours.

Conclusions Single gene targeted nanopore sequencing for diagnosing and simultaneously identifying key AMR markers for four common STIs shows promise. Further work to optimise accuracy, reduce costs and improve speed may allow sustainable approaches for managing STIs and emerging AMR in resource poor and laboratory limited settings.

\section{P361 VAGINAL MICROBIOME PROFILING IN INDIAN WOMEN WITH AND WITHOUT BACTERIAL VAGINOSIS}

${ }^{1} \mathrm{~A}$ Challa*, ${ }^{2} \mathrm{G}$ Kachhawa, ${ }^{3} \mathrm{~S}$ Sood, ${ }^{1} \mathrm{~S}$ Gupta. ${ }^{1}$ Department of Dermatology and Venereology, All India Institute of Medical Sciences, India; ${ }^{2}$ Department of Obstetrics and Gynaecology, All India Institute of Medical Sciences, India; ${ }^{3}$ Department of Microbiology, All India Institute of Medical Sciences, India

\subsection{6/sextrans-2021-sti.405}

Background Bacterial vaginosis (BV), a common condition among reproductive age women is associated with an imbalance of vaginal microbiota composition. It has been implicated in numerous adverse reproductive outcomes and increased risk of sexually transmitted infections. The vaginal ecology varies widely among women from various geographic and ethnic backgrounds. Our study aimed to explore the spectrum of bacterial communities in Indian women with and without BV and the association of individual species with Amsel's clinical diagnostic criteria.

Methods 16S rRNA gene PCR and V3-V4 sequencing were performed on vaginal swabs from 38 women with BV (mean age $=30.84$ years, Nugent score $\geq 7$ ) and 16 healthy controls (mean age $=28.25$ years, Nugent score $\leq 3)$. BV was assessed by Amsel's clinical criteria and confirmed by Nugent scoring of Gram stained vaginal smears. Taxonomic classification was performed using SILVA reference database.

Results The vaginal community composition of women with BV was highly heterogeneous and characterized by species diversity and richness. Women with BV harbored high concentrations of Gardnerella vaginalis ( $>99 \%$ women), Atopobium spp (81.6\%), Prevotella timonensis (73.7\%), Sneathia amnii (73.7\%) and Sneathia sanguinegens (63.2\%). On the contrary, in healthy women with no evident symptoms of vaginitis, Lactobacillus species dominated the vaginal flora wherein $\mathrm{L}$ iners and L gasseri were the two most frequently detected species. Interestingly, L iners was noted in all women, irrespective of their BV status. The presence of Atopobium spp., Sneathia 